\title{
On the Shannon Cipher System with a Capacity-Limited Key-Distribution Channel
}

\author{
Neri Merhav \\ Department of Electrical Engineering \\ Technion - Israel Institute of Technology \\ Haifa 32000, ISRAEL \\ merhav@ee.technion.ac.il
}

\begin{abstract}
We consider the Shannon cipher system in a setting where the secret key is delivered to the legitimate receiver via a channel with limited capacity. For this setting, we characterize the achievable region in the space of three figures of merit: the security (measured in terms of the equivocation), the compressibility of the cryptogram, and the distortion associated with the reconstruction of the plaintext source. Although lossy reconstruction of the plaintext does not rule out the option that the (noisy) decryption key would differ, to a certain extent, from the encryption key, we show, nevertheless, that the best strategy is to strive for perfect match between the two keys, by applying reliable channel coding to the key bits, and to control the distortion solely via ratedistortion coding of the plaintext source before the encryption. In this sense, our result has a flavor similar to that of the classical source-channel separation theorem. Some variations and extensions of this model are discussed as well.
\end{abstract}

Index Terms: Shannon cipher system, key distribution, encryption, cryptography, source-channel separation.

\section{Introduction}

In the classical Shannon-theoretic approach to cryptology (see, e.g., [6, [4, [10] and references therein), two assumptions are traditionally made. The first is that the reconstruction of the decrypted plaintext source at the legitimate receiver is distortion-free (or almost distortion-free), and the second, which is related, is that the encryption and the decryption units share identical copies of the same key. Yamamoto [11] has relaxed the first assumption and extended the theory of Shannon secrecy systems into a rate-distortion scenario, allowing lossy reconstruction at the legtimate receiver. 
In this correspondence, we examine also the second assumption. Referring to Fig. 1, we consider the case where the key is delivered to the legitimate receiver across a channel, which is cryptographically secure, but has limited capacity. For this setting, we characterize the achievable region in the space of three figures of merit: the security level (measured in terms of the equivocation), the compressibility of the cryptogram, and the distortion associated with the reconstruction of the plaintext source.

One conceptually simple approach to handle such a situation would be to apply a reliable channel code to the encryption key bits, at a rate below the capacity of the channel, and thereby obtain, with high probability, the exact copy of the transmitted key bits at the receiver side. With this approach, however, the effective key rate, and hence the security level in terms of the equivocation, is limited by the channel capacity. The question that naturally arises at this point, especially in the lossy reconstruction scenario, is whether this is the best one can do.

To sharpen the question, let us even assume that there is an unlimited reservoir of random key bits at the transmitter side, denoted $\boldsymbol{K}=\left(K_{1}, K_{2}, \ldots\right), K_{i} \in\{0,1\}, i=$ $1,2, \ldots$ Then, perhaps one might wish to use more key rate (somewhat above capacity) for encryption and thereby increase the security of the cryptogram at the expense of some distortion at the reconstruction, due to the unavoidable mismatch between the encryption and decryption keys. To explore this point, let us consider a few speculative strategies.

In the first strategy, one sends the key bits $\boldsymbol{K}$ across the channel uncodedly (assuming, for simplicity, that the channel has a binary input-output alphabet). Referring to Fig. 1, let us take then $N=n$ and $X_{i}=K_{i}, i=1,2, \ldots$ In this case, the noisy version of the key, obtained at the receiver side, $K_{i}^{\prime}=Y_{i}$, is of course somewhat different from the original key. However, since only lossy reconstruction of the plaintext is required at the receiver side, it may seem conceivable that a reasonably small difference between the keys at both ends could be managable and thus cause a reasonably small distortion in the reconstruction. This is relatively easy to have if the encryption of the source precedes compression, as proposed in 2]: One may apply, for example, a certain memoryless mapping from the key bit stream into a stream of symbols $Z_{1}, Z_{2}, \ldots$ taking (two of the) values in the alphabet of plaintext source, $\mathcal{U}$. Then assuming that $\mathcal{U}$ is a commutative group endowed with an addition operation $\oplus$ (e.g., addition modulo the alphabet size), one can create the enctypted sequence $U_{i}^{\prime}=U_{i} \oplus Z_{i}, i=1,2, \ldots$ and then compress the block $\left(U_{1}^{\prime}, \ldots, U_{n}^{\prime}\right)$ 
with $\left(K_{1}^{\prime}, \ldots, K_{n}^{\prime}\right)$ as side information at the receiver, using a Slepian-Wolf encoder [7] in the lossless case, or a Wyner-Ziv code [9] in the lossy case. Assuming, for simplicity, lossless compression, then upon decompressing the source at the receiver side and obtaining $\left(\tilde{U}_{1}, \ldots, \tilde{U}_{n}\right)$ (which is with high probability equal to $\left(U_{1}^{\prime}, \ldots, U_{n}^{\prime}\right)$ ), one 'subtracts' the noisy version of the key and obtain (with high probability) the reconstruction $V_{i}=U_{i}^{\prime} \ominus Z_{i}^{\prime}$, $i=1,2, \ldots$, where $Z_{i}^{\prime}$ is the corresponding noisy version of $Z_{i}$. Now, since $V_{i} \ominus U_{i}=Z_{i} \ominus Z_{i}^{\prime}$, for all $i$, then for a difference distortion measure $d\left(U_{i}, V_{i}\right)=\rho\left(V_{i} \ominus U_{i}\right)$, the distortion between $U_{i}$ and its reconstruction $V_{i}$ is identical to the distortion between the original key $Z_{i}$ and its noisy version $Z_{i}^{\prime}$.

A somewhat more sophisticated version of this scheme generates $Z_{1}, Z_{2}, \ldots$ from the key bits using a simulator of a certain (memoryless) process (see, e.g., [8] and references therein), and then applies a good source-channel code to encode $\left(Z_{1}, \ldots, Z_{n}\right)$ across the channel. The reconstructed version at the receiver side, $Z_{1}^{\prime}, Z_{2}^{\prime}, \ldots$, would then have the minimum possible distortion relative to $\left(Z_{1}, \ldots, Z_{n}\right)$, given by the distortion-rate function of $\left\{Z_{i}\right\}$ computed at the channel capacity, and therefore so would be also the distortion between $\left\{U_{i}\right\}$ and $\left\{V_{i}\right\}$. Moreover, there is an additional degree of freedom with regard to the choice of the probability law of $\left\{Z_{i}\right\}$ for trading off between the security, which is given by the entropy rate of $\left\{Z_{i}\right\}$, and the distortion, i.e., distortion-rate function of $\left\{Z_{i}\right\}$ computed at the channel capacity.

Another solution strategy may be based on the following point: Note that for the purpose of reliable transmission and decoding of the key bits across the channel, the cryptogram (denoted by $W^{m}$ in Fig. 1), which is a function of these key bits as well, may serve as useful side information at the decoder, unless it is statistically independent of these bits. Thus, one would speculate that it might be wise to allow some dependence between $W^{m}$ and $\boldsymbol{K}$ and thus sacrifice some compression performance at the benefit gaining performance in communicating the key across the channel. Let us assume that the bits of the key string $K^{m}=\left(K_{1}, \ldots, K_{m}\right)$ are XORed (added modulo 2$)$ with the bits of the compressed version of the source. Then, if the compression algorithm is designed in such a way such the bits of the compressed version of $U^{N}$ are not symmetric, then $W^{m}$ is correlated to $K^{m}$, and so $W^{m}$ can be viewed as a noisy version of $K^{m}$, which was transmitted uncodedly across a "parallel channel". In such a case, we can then think of the key bits as being encoded using a systematic code across the combined channel whose outputs are $W^{m}$ and $Y^{n}$ and 
the effective rate of this code is smaller than that over the original channel depicted in Fig. 1. Another way to look at this is the following: The key string $K^{m}$ can be compressed by a Slepian-Wolf encoder given $W^{m}$ (as side information at the decoder) before being channel coded, thus increasing the effective capacity by a factor given by the reciprocal of the conditional entropy of the key given the cryptogram.

We show in this correspondence that none of the ideas raised in the last four paragraphs, nor any other creative idea one may have, can work better than the first strategy we mentioned earlier, which is the following: At the lower part of the encoder of Fig. 1 (the "key encoder"), use a good channel code at rate below capacity, whose role is to reliably transmit a certain amount of key bits. At the upper block of the encoder of Fig. 1, first compress $U^{N}$ by an optimal rate-distortion code to obtain $N R(D)$ bits, where $R(D)$ is the rate-distortion function of $U^{N}$, and then encrypt the compressed bitstream with the same bits that are fed into the channel code. At the receiver, first decode the key bits from the channel output, and then use them to decrypt and decompress the source.

The result on the optimality of this scheme has a flavor similar to that of the classical source-channel separation theorem in three aspects: (i) There is a complete decoupling between source coding (for $U^{N}$ ) and channel coding (for the key bits) from the operative point of view as well as from the viewpoint of code design (unlike in the other strategies described above), (ii) the best possible strategy of controlling the distortion is only via rate-distortion coding, and (iii) the necessary and sufficient condition for perfect secrecy is $N R(D) \leq n C$, which is of the same form as the source-channel separation theorem.

The outline of this correspondence is as follows. In Section 2, we define notation conventions and give a formal definition of the problem. In Section 3, we state and prove the main result, and in Section 4, we discuss a few variations and extensions.

\section{Notation Conventions and Problem Definition}

We begin by establishing some notation conventions. Throughout this paper, scalar random variables (RV's) will be denoted by capital letters, their sample values will be denoted by the respective lower case letters, and their alphabets will be denoted by the respective calligraphic letters. A similar convention will apply to random vectors and their sample values, which will be denoted with same symbols superscripted by the dimension. Thus, for example, $U^{N}(N$ - positive integer $)$ will denote a random $N$-vector $\left(U_{1}, \ldots, U_{N}\right)$, and 
$u^{N}=\left(u_{1}, \ldots, u_{N}\right)$ is a specific vector value in $\mathcal{U}^{N}$, the $N$-th Cartesian power of $\mathcal{U}$.

Sources and channels will be denoted generically by the letter $P$, subscripted by the name of the RV and its conditioning, if applicable, e.g., $P_{U}(u)$ is the probability function of $U$ at the point $U=u, P_{Y \mid X}(y \mid x)$ is the conditional probability of $Y=y$ given $X=x$, and so on. Whenever clear from the context, these subscripts will be omitted. Information theoretic quantities like entropies and mutual informations will be denoted following the usual conventions of the Information Theory literature, e.g., $H\left(U^{N}\right), I\left(X^{n} ; Y^{n}\right)$, and so on. For single-letter information quantities (i.e., when $n=1$ or $N=1$ ), subscripts will be omitted, e.g., $H\left(U^{1}\right)=H\left(U_{1}\right)$ will be denoted by $H(U)$, similarly, $I\left(X^{1} ; Y^{1}\right)=I\left(X_{1} ; Y_{1}\right)$ will be denoted by $I(X ; Y)$, and so on.

We now turn to the formal description of the model and the problem setting, as described in the Introduction, and referring to Fig. 1. A source $P_{U}$, generates a sequence of independent copies, $U_{1}, U_{2}, \ldots$ of a finite-alphabet RV, $U \in \mathcal{U}$, whose entropy is $H(U) \triangleq-\sum_{u \in \mathcal{U}} P_{U}(u) \log _{2} P_{U}(u)$. At the same time and independently, a discrete memoryless channel (DMC) $P_{Y \mid X}$ receives input symbols $x_{1}, x_{2}, \ldots$ with coordinates taking values in a finite alphabet $\mathcal{X}$, and produces output symbols $y_{1}, y_{2}, \ldots$ with coordinates taking values in a finite alphabet $\mathcal{Y}$, according to a conditional probability law given by the product of single-letter transition probabilities $\prod_{t} P_{Y \mid X}\left(y_{t} \mid x_{t}\right)$. The relative rate between the operation of the channel $P_{Y \mid X}$ and that of the source is $\lambda$ channel symbols per source symbol. This means that while the source generates a block of $N$ symbols, say, $U^{N}=\left(U_{1}, \ldots, U_{N}\right)$, according to the above mentioned probability law, the channel conveys $n=\lambda N$ transmissions, ${ }^{1}$ i.e., it receives a channel input block of length $n, X^{n}=\left(X_{1}, \ldots, X_{n}\right)$, and outputs another block of the same length $Y^{n}=\left(Y_{1}, \ldots, Y^{n}\right)$ according to the above described conditional probability law. Let $C=\max _{P_{X}} I(X ; Y)$ denote the channel capacity.

In addition to the source $P_{U}$ and the channel $P_{Y \mid X}$, yet another source, $P_{K}$, henceforth referred to as the key source, generates an infinite sequence of i.i.d. purely random bits, $\boldsymbol{K}=\left(K_{1}, K_{2}, \ldots\right)$, independently of the source $U_{1}, U_{2}, \ldots$ The operation rate of the key source relative to the source $P_{U}$ (and the channel $P_{Y \mid X}$ ) will be immaterial, i.e., we will assume that the reservoir of key bits, for every finite period of time, is sufficiently large so that it is effectively unlimited.

A block code for joint coding and encryption with parameters $n$ and $\lambda=n / N$, consists

\footnotetext{
${ }^{1}$ Without essential loss of generality, we will assume that $\lambda N$ is a positive integer.
} 
of three mappings. The first mapping is the compressor-encrypter $f_{N}: \mathcal{U}^{N} \times\{0,1\}^{\infty} \rightarrow$ $\{0,1\}^{m}$, where $m=\mu N, \mu>0$ being the compression rate. Upon receiving a source vector $u^{N} \in \mathcal{U}^{N}$ and a key sequence $\boldsymbol{k} \in\{0,1\}^{\infty}$, this mapping produces a binary cryptogram $w^{m} \in\{0,1\}^{m}$ according to $w^{m}=f_{N}\left(u^{N}, \boldsymbol{k}\right)$. The second mapping is the key-encoder $g_{N}$ : $\{0,1\}^{\infty} \rightarrow \mathcal{X}^{n}$, which produces a channel input vector $x^{n}$ according to $x^{n}=g_{n}(\boldsymbol{k})$. Finally, the third mapping is the decoder $h_{N}:\{0,1\}^{m} \times \mathcal{Y}^{n} \rightarrow \mathcal{V}^{N}$, where $\mathcal{V}$ is the reproduction alphabet. Upon receiving a cryptogram $w^{m}$ and a channel output vector $y^{n}$, the decoder produces a reproduction vector according to $v^{N}=h_{N}\left(w^{m}, y^{n}\right)$.

Let $d: \mathcal{U} \times \mathcal{V} \rightarrow \mathbb{R}^{+}$denote a single-letter distortion measure between source symbols and the reproduction symbols, and let the distortion between the vectors, $u^{N} \in \mathcal{U}^{N}$ and $v^{N} \in \mathcal{V}^{N}$, be defined additively across the corresponding components, as usual. We will assume that $d$ is bounded, i.e., $d_{\max } \triangleq \max _{u, v} d(u, v)<\infty$. Let $R(D)$ denote the ratedistortion function of the source $P_{U}$ with respect to $d$.

An $\left(n, \lambda, D, R_{c}, h\right)$ code for joint coding and encryption is a block code with parameters $n$ and $\lambda$, as above, which also satisfies the following requirements:

1. The expected distortion between the source and the reproduction satisfies

$$
\sum_{i=1}^{N} E d\left(U_{i}, V_{i}\right) \leq N D .
$$

2. The rate of the cryptogram satisfies

$$
\mu=\frac{m}{N} \leq R_{c}
$$

3. The equivocation of the source satisfies

$$
H\left(U^{N} \mid W^{m}\right) \geq N h
$$

For a given $\lambda$, a triple $\left(D, R_{c}, h\right)$ is said to be achievable if for every $\epsilon>0$, there is a sufficiently large $n$ for which $\left(n, \lambda, D+\epsilon, R_{c}+\epsilon, h-\epsilon\right)$ block codes for joint coding and encryption exist. Our purpose, in this paper is to characterize the achievable region of triples $\left(D, R_{c}, h\right)$, i.e., the set of all achievable triples $\left(D, R_{c}, h\right)$.

\section{Main Result}

Our main coding theorem is the following: 
Theorem 1 A triple $\left(D, R_{c}, h\right)$ is achievable if and only if the following conditions are both satisfied:

(a) $h \leq h^{*}(D) \triangleq H(U)-[R(D)-\lambda C]_{+}$, where $[a]_{+} \triangleq \max \{a, 0\}$.

(b) $R_{c} \geq R(D)$.

It should be noted that for a given $D$, there is no conflict (or interaction) between maximizing $h$ and minimizing $R_{c}$ : As is well known, $R_{c}$ is lower bounded by $R(D)$ even if there is no security requirement, but on the other hand, even in the presence of the highest possible security level requirement, of $h^{*}(D)$, the compression ratio $R(D)$ is still achievable [1]. By the same token, and as will be evident from the proof, $h$ is upper bounded by $h^{*}(D)$ even if there is no compressibility requirement, yet it remains achievable even if the compression ratio of $R(D)$ is required.

The remaining part of this section is devoted to the proof of Theorem 1 .

Proof. We begin with the converse part. Let an $\left(n, \lambda, D+\epsilon, R_{c}+\epsilon, h-\epsilon\right)$ block code for joint coding and encryption be given. Now, since

$$
h^{*}(D)=H(U)-[R(D)-\lambda C]_{+}=\min \{H(U), H(U)-R(D)+\lambda C\},
$$

we have to prove that both $h \leq H(U)$ and $h \leq H(U)-R(D)+\lambda C$. The first bound is trivial since

$$
N(h-\epsilon) \leq H\left(U^{N} \mid W^{m}\right) \leq H\left(U^{N}\right)=N H(U)
$$

where the first inequality is by definition of an $\left(n, \lambda, D+\epsilon, R_{c}+\epsilon, h-\epsilon\right)$ block code for joint coding and encryption. The inequality $h \leq H(U)$ now follows from the arbitrariness of $\epsilon>0$. As for the second bound, we have

$$
\begin{aligned}
N(h-\epsilon) & \leq H\left(U^{N} \mid W^{m}\right) \\
& =H\left(U^{N} \mid W^{m}, Y^{n}\right)+I\left(U^{N} ; Y^{n} \mid W^{m}\right) \\
& =H\left(U^{N} \mid W^{m}, Y^{n}, V^{N}\right)+H\left(Y^{n} \mid W^{m}\right)-H\left(Y^{n} \mid W^{m}, U^{N}\right) \\
& \leq H\left(U^{N} \mid V^{N}\right)+H\left(Y^{n}\right)-H\left(Y^{n} \mid W^{m}, U^{N}, X^{n}\right) \\
& =H\left(U^{N}\right)-I\left(U^{N} ; V^{N}\right)+H\left(Y^{n}\right)-H\left(Y^{n} \mid X^{n}\right) \\
& \leq N H(U)-N R(D+\epsilon)+I\left(X^{n} ; Y^{n}\right) \\
& \leq N[H(U)-R(D+\epsilon)]+n C,
\end{aligned}
$$


where the second line is a standard identity, the third is because $V^{N}$ is a function of $\left(W^{m}, Y^{n}\right)$, the fourth is because conditioning reduces entropy (used thrice), the fifth is due to the fact that $\left(U^{N}, W^{m}\right) \rightarrow X^{n} \rightarrow Y^{n}$ is a Markov chain, the sixth is due to the memorylessness of the source and the fact that $R(D)=\min \{I(U ; V): \operatorname{Ed}(U, V) \leq D\}$ (which is also convex), and the last line is due to the memorylessness of the channel and the fact that $C=\max _{P_{X}} I(X ; Y)$. Again, dividing by $N$, and using the arbitrariness of $\epsilon>0$ as well as the continuity of $R(D)$, we get the second bound on $h$, and so, the necessity of condition (a) follows.

The proof of the necessity of condition (b) is similar to the proof of the converse to the ordinary rate-distortion coding theorem, except that the presence of $Y^{n}$ (which is independent of $U^{N}$ ) at the decoder has to be taken into account:

$$
\begin{aligned}
N\left(R_{c}+\epsilon\right) & \geq H\left(W^{m}\right) \\
& \geq H\left(W^{m} \mid Y^{n}\right) \\
& \geq I\left(U^{N} ; W^{m} \mid Y^{n}\right) \\
& =\sum_{i=1}^{N}\left[H\left(U_{i} \mid U^{i-1}, Y^{n}\right)-H\left(U_{i} \mid U^{i-1}, W^{m}, Y^{n}\right)\right] \\
& \geq \sum_{i=1}^{N}\left[H\left(U_{i}\right)-H\left(U_{i} \mid W^{m}, Y^{n}\right)\right] \\
& =\sum_{i=1}^{N} I\left(U_{i} ; W^{m}, Y^{n}\right) \\
& \geq \sum_{i=1}^{N} I\left(U_{i} ; V_{i}\right) \\
& \geq N R(D+\epsilon),
\end{aligned}
$$

where the first line is by definition of an $\left(n, \lambda, D+\epsilon, R_{c}+\epsilon, h-\epsilon\right)$ block code for joint coding and encryption, the second, third, fourth and sixth are standard identities and inequalities, the fifth is based on the memorylessness of the source and its independence of $Y^{n}$, the seventh is based on the data processing inequality and the fact that $V_{i}$ is a function of $\left(W^{m}, Y^{N}\right)$, and the last inequality is again by the informational definition of $R(D)$ and its convexity. Taking again $\epsilon$ to zero, this completes the proof of the converse part of Theorem 1.

As for the direct part, consider the following (conceptually) simple coding scheme. For a given arbirarily small $\epsilon>0$, let $\ell=\min \{n(C-\epsilon), N[R(D)+\epsilon]\}$ and let $x^{n}=g_{N}\left(k_{1}, \ldots, k_{\ell}\right)$ 
be given by a channel code whose error probability is below some $\delta>0$, provided that $n$ is sufficiently large. Since the rate of this code never exceeds $C-\epsilon$, such a channel code exists by the classical channel coding theorem. As for $f_{N}$, first apply a rate-distortion code for $U^{N}$, whose rate is $R_{c}=R(D)+\epsilon$, and then encrypt $\ell$ of the resulting $m=N[R(D)+\epsilon]$ bits by $\left(k_{1}, \ldots, k_{\ell}\right)$ (using the ordinary bit-by-bit XOR). As for the equivocation, we have

$$
\begin{aligned}
H\left(U^{N} \mid W^{m}\right) & =H\left(U^{N}\right)-I\left(U^{N} ; W^{m}\right) \\
& =N H(U)-H\left(W^{m}\right)+H\left(W^{m} \mid U^{N}\right) \\
& \geq N H(U)-N[R(D)+\epsilon]+H\left(W^{m} \mid U^{N}\right) \\
& =N H(U)-N[R(D)+\epsilon]+\ell \\
& =N H(U)-N[R(D)+\epsilon]+\min \{n(C-\epsilon), N[R(D)+\epsilon]\} \\
& \geq N\left(H(U)-[R(D)-\lambda C]_{+}-2 \epsilon \max \{1, \lambda\}\right),
\end{aligned}
$$

where the first inequality follows from the fact that the rate-distortion code is at rate $R(D)+\epsilon$, and the following equality is due to the fact that $\ell$ bits of the compressed bit string are encrypted. At the decoder, first, the $\ell$ key bits $\left(k_{1}, \ldots, k_{\ell}\right)$ are decoded, and then

the decoded key bits $\left(\hat{k}_{1}, \ldots, \hat{k}_{\ell}\right)$ are used to decrypt $w^{m}$ and then use the rate-distortion decoder to produce $v^{N}$. With probability at least $1-\delta$, the decoded key bits $\left(\hat{k}_{1}, \ldots, \hat{k}_{\ell}\right)$ agree with the original ones $\left(k_{1}, \ldots, k_{\ell}\right)$ and then $w^{m}$ is decrypted correctly to produce the appropriate reproduction vector $v^{N}$ within distortion $D$. At the event of erroneous decoding of $\left(k_{1}, \ldots, k_{\ell}\right)$, the distortion can only be bounded by $d_{\max }$, but this should be weighed by the probability of error, which is upper bounded by $\delta$, and hence contributes only an arbitrarily small additional distortion. This completes the proof of Theorem 1.

\section{Discussion}

In this section, we discuss a few variations and extensions of the model considered.

\subsection{Source-Channel Separation}

We have already mentioned in the Introduction that Theorem 1 has the spirit of a separation theorem, from several points of view. Among them is the immediate observation that perfect security (in the sense that $h=H(U)$ ) can be achieved if and only if $R(D) \leq \lambda C$, an inequality of the very same form as that of the classical joint source-channel separation 
theorem. In this context, we should also point out that it is straightforward to extend our setup to a situation of ordinary joint source-channel coding, corresponding to the case where the cryptogram $w^{m}$ needs to be transmitted via a noisy channel, independent of the key distribution channel. The only modification to Theorem 1 would be to replace $R_{c}$ in part (b) by the capacity of the main channel. Thus, we have a two-fold separation theorem.

\subsection{Simple Coding and Decoding in Special Cases}

Suppose that the compressibility of the cryptogram is not an issue, in other words, $R_{c}$ is immaterial and we are only interested in the tradeoff between $D$ and $h$. In this case, there exist situations where optimal performance can be achieved using very simple coding systems, similiarly to the well-known special cases, where this can be done in the context of classical joint source-channel coding (see, e.g., [1]). Let us suppose, for example, that $\mathcal{U}=\mathcal{X}=\mathcal{Y}=\mathcal{V}, \lambda=1$, and that the distortion measure $d$ is a difference distortion measure, i.e., $d(u, v)=\rho(v \ominus u)$ for a well-defined subtraction operation (cf. the corresponding discussion in the Introduction). Suppose further that $P_{U}$, which is the uniform distribution over $\mathcal{U}$, is the capacity-achieving input for the channel $P_{Y \mid X}$ and that $P_{Y \mid X}$ in turn achieves the rate-distortion function of $P_{U}$ at distortion level $D$, i.e., $R(D)=C$. For example, $P_{U}$ may be the BSS and $P_{Y \mid X}$ may be the BSC with crossover probability $D$. Then one can easily achieve perfect secrecy, $h=H(U)=\log |\mathcal{U}|$, at the minimum possible distortion, i.e., $D=R^{-1}(C)\left(R^{-1}(\cdot)\right.$ being the distortion-rate function of $\left.U\right)$ in the following manner, which is similar to one of the strategies discussed in the Introduction: Let $Z_{1}, Z_{2}, \ldots$ be a simulated memoryless process, generated from $\boldsymbol{K}$, with the same (uniform) distribution as $U_{1}, U_{2}, \ldots$. Note that when $|\mathcal{U}|$ is a power of 2 , this is very easy to implement since $U$ is uniform. For encryption, let $W_{i}=U_{i} \oplus Z_{i}$. Then, obviously, $H\left(U^{N} \mid W^{N}\right)=N H(U)=N \log |\mathcal{U}|$ since $U_{i}$ and $Z_{i}$ are uniformly distributed and independent, and so, perfect secrecy is guaranteed. As for the key transmission, let us send $\left\{Z_{i}\right\}$ uncodedly across the channel, i.e., $X_{i}=Z_{i}$. Since $P_{Y \mid X}$ achieves the rate-distortion of $\left\{U_{i}\right\}$, and hence also that of $\left\{Z_{i}\right\}$, then the channel output $\left\{Y_{i}\right\}$ will have distortion $D$ relative to $\left\{Z_{i}\right\}$. At the the decoder, we simply apply the equation $V_{i}=W_{i} \ominus Y_{i}$. Since $V_{i} \ominus U_{i}=Z_{i} \ominus Y_{i}$, then $\operatorname{Ed}\left(U_{i}, V_{i}\right)=E \rho\left(V_{i} \ominus U_{i}\right)=$ $E \rho\left(Z_{i} \ominus Y_{i}\right)=D=R^{-1}(C)$. Thus, optimal performance is achieved using a very simple system once we have an independent copy of $\left\{U_{i}\right\}$ as a key. 


\subsection{A Wider Class of Joint Encoders}

Another point regarding the case where $R_{c}$ is immaterial, is the following: It turns out that part (a) of Theorem 1 (both the necessity and the sufficiency) would still apply even if we broaden the scope to a wider class of encoders that allow both $x^{n}$ and $w^{m}$ to depend on both $u^{N}$ and $\boldsymbol{k}$. This means that $g_{N}$ is redefined as $g_{N}:\{0,1\}^{\infty} \times \mathcal{U}^{N} \rightarrow \mathcal{X}^{n}$, and so, $x^{n}=g_{N}\left(\boldsymbol{k}, u^{N}\right)$. The direct part would use the same scheme as before. As for the converse part, note that eq. (6) is general enough to allow this setup. The conclusion then is that if only $D$ and $h$ are the figure of merits of interest, then a good key code $g_{N}$ need not really use its accessibility to $u^{N}$. The situation becomes somewhat more involved when the compressibility is brought back into the picture, because then the encoder has two paths through which it can pass descriptions of the source. Note that if $R(D) \leq \lambda C$, the encoder can transmit the entire description via the key distribution channel, without using the main channel at all, thus $R_{c}=0$.

\subsection{Securing the Reproduction Sequence}

Consider the case where one is interested not only to guarantee a certain security level $h$ with regard to the original source, but also to guarantee a security level $h^{\prime}$ with regard to the reproduction $V^{N}$. This makes sense because it is actually $V^{N}$ the part of the information that is communicated to the legitimate receiver and thus has to be protected (see also [5]). To derive necessary conditions for securing $V^{N}$ at level $h^{\prime}$, we consider two chains of inequalities. The first is the following:

$$
\begin{aligned}
N\left(h^{\prime}-\epsilon\right) & \leq H\left(V^{N} \mid W^{m}\right) \\
& \leq H\left(V^{N}\right) \\
& \leq \sum_{i=1}^{N} H\left(V_{i}\right) \\
& =N H(V \mid J) \\
& \leq N H(V)
\end{aligned}
$$

where $J$ is random variable taking values in the set $\{1, \ldots, N\}$ with the uniform distribution

and $V \triangleq V_{J}$. Thus, our first necessary condition for security level $h^{\prime}$ is that there exists a random variable $V$ with alphabet $\mathcal{V}$ (jointly distributed with $U$ ) such that $h^{\prime} \leq H(V)$. The 
second chain of inequalities is as follows:

$$
\begin{aligned}
N\left(h^{\prime}-\epsilon\right) & \leq H\left(V^{N} \mid W^{m}\right) \\
& =H\left(V^{N} \mid W^{m}, Y^{n}\right)+I\left(Y^{n} ; V^{N} \mid W^{m}\right) \\
& =I\left(Y^{n} ; V^{N} \mid W^{m}\right) \\
& \leq H\left(Y^{n} \mid W^{m}\right) \\
& \leq H\left(Y^{n}\right) \\
& \leq \sum_{i=1}^{n} H\left(Y_{i}\right) \\
& =n H\left(Y \mid J^{\prime}\right) \\
& \leq n H(Y),
\end{aligned}
$$

where $J^{\prime}$ is random variable taking values in the set $\{1, \ldots, n\}$ with the uniform distribution

and $Y \triangleq Y_{J^{\prime}}$. The second equality is due to the fact that $V^{N}$ is a function of $\left(W^{m}, Y^{n}\right)$ and so $H\left(V^{N} \mid W^{m}, Y^{n}\right)=0$. Thus, another necessary condition is the existence of random variable $Y$ at the output of the channel $P_{Y \mid X}$ (which means the existence of a channel input variable $X$ that induces $Y$ via $\left.P_{Y \mid X}\right)$ such that $h^{\prime} \leq \lambda H(Y)$. The combination of the two necessary conditions then gives $h^{\prime} \leq \min \{H(V), \lambda H(Y)\}$.

A restatement of the necessity part of Theorem 1 would then be the following: If $\left(D, R_{c}, h, h^{\prime}\right)$ is achievable then there exist a channel $P_{V \mid U}$ and a source $P_{X}$ such that the following conditions are simultaneously satisfied:

(a) $h \leq H(U)-[I(U ; V)-\lambda I(X ; Y)]_{+}$,

(b) $h^{\prime} \leq \min \{H(V), \lambda H(Y)\}$,

(c) $R_{c} \geq I(U ; V)$

(d) $D \geq E d(U, V)$.

Note that in contrast to Theorem 1, we are no longer taking the minimum of $I(U ; V)$ to obtain $R(D)$, nor do we take the maximum of $I(X ; Y)$ to obtain $C$. The reason is that such optimizations might be in partial conflict with the need to achieve large values of $H(V)$ and $H(Y)$ in order to meet condition (b). Thus, there are more complicated compromises in the choice of $X$ and $V$ when the tradeoff involves the additional parameter $h^{\prime}$. 
The achievability of this set of conditions remains open in general. However, for the special case where the channel $P_{Y \mid X}$ is deterministic, that is, $Y$ is a deterministic function of $X$, and so $I(X ; Y)=H(Y)$, the achievability scheme is essentially the same as before (but with general choices of $P_{X}$ and $\left.P_{V \mid U}\right)$ as long as the required security $h^{\prime}$ does not exceed the level $\min \{I(U ; V), \lambda I(X ; Y)\}=\min \{I(U ; V), \lambda H(Y)\}$. If it is higher, and if $\lambda H(Y)$ exceeds $I(U ; V)$ the additional key bits beyond $N I(U ; V)$ (but not more than $H(V)$ ) conveyed by the channel can be used to control the (secret) choice of the rate-distortion codebook among up to $2^{N H(V \mid U)}$ distinct codebooks that exist (cf. [3], [5]) and thereby achieve the extra security needed with regard to $V^{N}$.

Note that here, the separation principle no longer holds as before, in the strong meaning of this term, because now, the choice of $P_{X}$ and $P_{V \mid U}$ involves compromises where there is an interaction between the source coding of $U^{N}$ and the channel coding of $\boldsymbol{K}$.

\subsection{Feedback}

Finally, consider the scenario of the previous subsection, where in addition, there is noiseless feedback from the channel output to the transmitter. In this case, it is clear too how to secure $V^{N}$ to the level of $h^{\prime}=\min \{H(V), \lambda H(Y)\}$, and it is also clear that this value cannot be further improved upon. Here, the encoder and the decoder simply share identical copies of $\left\{Y_{i}\right\}$ as a common key at both ends, and there is no longer use for the original key, $\left\{K_{i}\right\}$. By the same token, in this case, the equivocation of $U^{N}$ can be enhanced to the level of $h=H(U)-[I(U ; V)-\lambda H(Y)]_{+}$, but not more. Thus, although feedback does not increase the capacity of a DMC, it certainly improves its effectiveness when this channel serves for key delivery.

\subsection{Continuous Alphabets}

In our derivations this far, we have limited ourselves to finite alphabet sources and channels, primarily for reasons of convenience. Theorem 1 extends quite straightforwardly to the continuous alphabet case as well. One comment is in order, however: In the continuous alphabet case, it no longer makes sense to measure equivocation in terms of conditional (differential) entropy, which can be negative. It still makes sense, nonetheless, to define it by the complementary quantity - the mutual information, $I\left(W^{m} ; U^{N}\right)$, which is always non-negative. Thus, part (a) of Theorem 1 would be restated to assert that $[R(D)-\lambda C]_{+}$ 
is an achievable lower bound to $I\left(W^{m} ; U^{N}\right) / N$.

\section{References}

[1] M. Gastpar, "To code or not to code," D.Sc. dissertation, EPFL, Lausanne, Switzerland, 2003.

[2] M. Johnson, P. Ishwar, V. Prabhakan, D. Schonberg, and K. Ramchandran, "On compressing encrypted data," IEEE Trans. Signal Processing, vol. 52, no. 10, pp. 2992-3006, October 2004.

[3] A. Maor and N. Merhav, "On joint information embedding and lossy compression," to appear in IEEE Trans. Inform. Theory.

[4] J. L. Massey, "An introduction to contemporary cryptology," Proc. IEEE, vol. 26, no. 5, pp. 533-549, May 1988.

[5] N. Merhav, "on joint coding for watermarking and encryption," submitted to IEEE Trans. Inform. Theory. Also, available on-line at http://www.ee.technion.ac.il/people/merhav.

[6] C. E. Shannon, "Communication theory of secrecy systems," Bell Syst. Tech. J., vol. 28, no. 3, pp. 565-715, Oct. 1949.

[7] D. Slepian and J. K. Wolf, "Noiseless coding of correlated information sources," IEEE Trans. Inform. Theory, vol. IT-19, pp. 471-480, 1973.

[8] Y. Steinberg and S. Verdú, "Simulation of random processes and rate-distortion theory," IEEE Trans. Inform. Theory, vol. 42, no. 1, pp. 63-86, January 1996.

[9] A. D. Wyner and J. Ziv, "The rate-distortion function for source coding with side information at the decoder," IEEE Trans. Inform. Theory, vol. IT-22, no. 1, pp. 1-10, January 1976.

[10] H. Yamamoto, "Information theory in cryptology," IEICE Trans., vol. E 74, no. 9, pp. 2456-2464, September 1991.

[11] H. Yamamoto, "Rate-distortion theory for the Shannon cipher system," IEEE Trans. Inform. Theory, vol. IT-43, pp. 827-835, May 1997. 


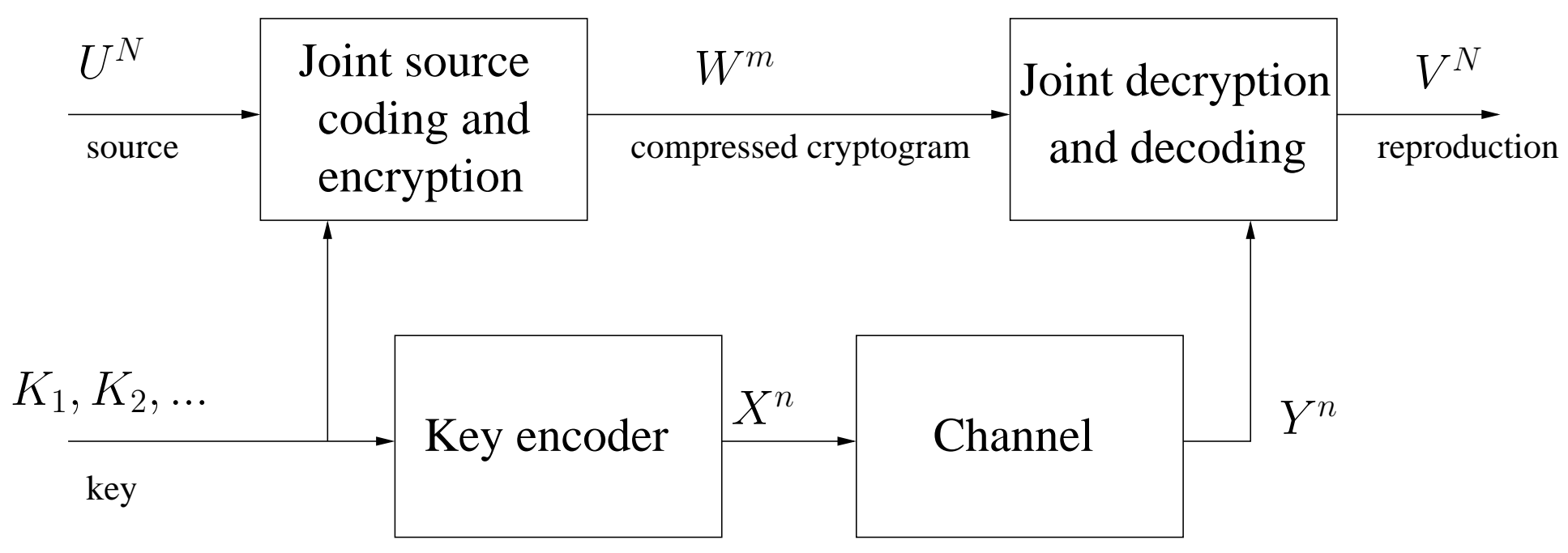

Figure 1: A cipher system with capacity-limited key distribution. 